\title{
Prevention of Type II diabetes in subjects with impaired glucose tolerance: the Diabetes Prevention Study (DPS) in Finland
}

\section{Study design and 1-year interim report on the feasibility of the lifestyle intervention programme}

\author{
J. Eriksson ${ }^{1}$, J. Lindström ${ }^{1}$, T. Valle ${ }^{1}$, S. Aunola ${ }^{2}$, H. Hämäläinen ${ }^{2}$, P. Ilanne-Parikka ${ }^{3}$, S. Keinänen-Kiukaanniemi ${ }^{4}$, \\ M. Laakso ${ }^{4}$, M. Lauhkonen ${ }^{5}$, P. Lehto ${ }^{5}$, A. Lehtonen ${ }^{6}$, A. Louheranta ${ }^{7}$, M. Mannelin ${ }^{8}$, V. Martikkala ${ }^{8}$, M. Rastas $^{2}$, \\ J. Sundvall ${ }^{9}$, A. Turpeinen ${ }^{7}$, T. Viljanen ${ }^{10}$, M. Uusitupa ${ }^{7}$, J. Tuomilehto ${ }^{1}$ on behalf of the Finnish Diabetes Prevention \\ Study Group \\ ${ }^{1}$ National Public Health Institute, Department of Epidemiology and Health Promotion, Diabetes and Genetic Epidemiology Unit, \\ Helsinki, Finland \\ ${ }^{2}$ Social Insurance Institution, Research and Development Centre, Turku, Finland \\ ${ }^{3}$ Finnish Diabetes Association, The Diabetes Centre, Tampere, Finland \\ ${ }^{4}$ University of Oulu, Department of Public Health Science and General Practice, Oulu, Finland \\ ${ }^{5}$ Institute of Nursing and Health Care, Tampere, Finland \\ ${ }^{6}$ University of Turku, Department of Geriatrics, Turku, Finland \\ ${ }^{7}$ University of Kuopio, Department of Clinical Nutrition, Kuopio, Finland \\ ${ }^{8}$ Department of Sports Medicine, Oulu Deaconess Institute, Oulu, Finland \\ ${ }^{9}$ National Public Health Institute, Department of Biochemistry, Helsinki, Finland \\ ${ }^{10}$ Paavo Nurmi Centre, Turku, Finland
}

\section{Abstract}

Aims/hypothesis. The aim of the Diabetes Prevention Study is to assess the efficacy of an intensive diet-exercise programme in preventing or delaying Type II (non-insulin-dependent) diabetes mellitus in subjects with impaired glucose tolerance, to evaluate the effects of the intervention programme on cardiovascular risk factors and to assess the determinants for the progression to diabetes in persons with impaired glucose tolerance.

Methods. A total of 523 overweight subjects with impaired glucose tolerance ascertained by two oral glucose tolerance tests were randomised to either a control or intervention group. The control subjects received general information at the start of the trial about the lifestyle changes necessary to prevent diabetes and about annual follow-up visits. The intervention subjects had seven sessions with a nutritionist during the first year and a visit every 3 months thereafter aimed at reducing weight, the intake of saturat- ed fat and increasing the intake of dietary fibre. Intervention subjects were also guided individually to increase their physical activity.

Results. During the first year, weight loss in the first 212 study subjects was $4.7 \pm 5.5$ vs $0.9 \pm 4.1 \mathrm{~kg}$ in the intervention and control group, respectively $(p<0.001)$. The plasma glucose concentrations (fasting: $5.9 \pm 0.7$ vs $6.4 \pm 0.8 \mathrm{mmol} / 1, p<0.001$; and 2 -h $7.8 \pm 1.8$ vs $8.5 \pm 2.3 \mathrm{mmol} / \mathrm{l}, p<0.05)$ were significantly lower in the intervention group after the first year of intervention. Favourable changes were also found in blood pressure, serum lipids and anthropometric indices in the intervention group.

Conclusion/interpretation. The interim results show the efficacy and feasibility of the lifestyle intervention programme. [Diabetologia (1999) 42: 793-801]

Keywords Type II diabetes mellitus, impaired glucose tolerance, diet, physical activity, primary prevention, lipids, weight, obesity, glucose, blood pressure.
Received: 7 December 1998 and in revised form: 23 February 1999

Corresponding author: Dr. J. Eriksson, National Public Health Institute, Department of Epidemiology and Health Promotion, Mannerheimintie 166, SF-00300 Helsinki, Finland
Abbreviations: IGT, Impaired glucose tolerance; WHO, World Health Organisation; DPS, Diabetes Prevention Study; CVD, cardiovascular disease; VLCD, very low calorie diet; HLA, human leukocyte antigen; GAD, glutamic acid decarboxylase. 
Type II (non-insulin-dependent) diabetes mellitus is an increasingly common disease with a large impact on society. The disease is characterised by the development of both microvascular and macrovascular complications. These complications, in particular atherosclerotic vascular disease, account for most of the increased morbidity and mortality associated with Type II diabetes [1-5]. In addition to the human suffering, the costs of diabetes care are rising rapidly worldwide [6-7] and are mostly attributed to the long-term complications. Unfortunately, there are no effective methods available to prevent these complications and the treatment options for Type II diabetes are also limited. Good glycaemic control, however, as well as good control of blood pressure has been shown to reduce the incidence of long-term complications $[8,9]$.

It has been estimated that worldwide the number of diabetic patients will more than double during the next 15 years and in Europe their number will increase from approximately 16 million in 1994 to 24 million in 2010 [10]. The prevention of Type II diabetes has become a major issue both scientifically and from a public health point of view [11]. Type II diabetes has a strong genetic component and hyperglycaemia usually worsens with time $[11,12]$. When genetically predisposed subjects become more insulin resistant, for instance due to obesity and physical inactivity, they could develop postprandial hyperglycaemia or impaired glucose tolerance (IGT) [13, 14]. When beta-cell capacity is no longer sufficient to compensate for the insulin resistance, hyperglycaemia worsens and overt diabetes develops. Thus, IGT can be viewed as a subclinical disease state with an increased risk of cardiovascular morbidity and mortality [15-17]. Furthermore, IGT is the first abnormal stage in the natural history of Type II diabetes that can be identified quite easily. Therefore, subjects with IGT can be a target for intervention and are suitable for testing the feasibility and effectiveness of the primary prevention of Type II diabetes. The overall prevalence of IGT varies greatly between studies, depending on the study population and the survey methods used. In the 45-54 year age group the prevalence varies between 2.0 and $13.6 \%$ in European populations, and for the age group over 65 years the corresponding figures are $6.8-23 \%$ [18]. Subjects with IGT have an increased risk of developing diabetes with a decompensation rate of $1-10 \%$ per year, and the cumulative incidence is as high as $50 \%$ [18].

Even though the primary prevention of diabetes was first proposed more than 70 years ago [19], and more recently stressed by the World Health Organisation (WHO) [20], there are only a few previous studies to assess the value of measures aimed at reducing obesity and increasing physical activity in people with IGT [21-28]. Several of the previous intervention studies used drugs, not only lifestyle inter- ventions. Unfortunately none of the previous lifestyle intervention studies have been properly designed to give a definite answer to the question of whether primary prevention of Type II diabetes is possible, but they have provided suggestive evidence for this. Moreover, the increased risk for individuals with IGT of developing frank diabetes and also macrovascular disease certainly justifies an intervention study in this high-risk group.

The main aim of the Finnish Diabetes Prevention Study (DPS) is to assess the efficacy of an intensive and individually designed diet and exercise programme to prevent or delay the onset of Type II diabetes in Finnish subjects with IGT. In addition, the DPS will determine factors associated with the progression to diabetes in persons with IGT. Since the intensive lifestyle intervention will probably also have beneficial effects on other risk factors for cardiovascular disease (CVD), we will assess the effects of the intervention on CVD risk factors and on the composite risk factor score for CVD.

The purpose of this paper is to describe the study design of the DPS. In addition, we will report data from the first interim analysis aimed at evaluating the feasibility of the study protocol during the first year of the study.

\section{Subjects and methods}

Study design. The study protocol was approved by the ethics committee of the National Public Health Institute in Helsinki, Finland.

The DPS is a multicentre study with five participating centres in Finland, located in Helsinki, Kuopio, Oulu, Tampere and Turku. Each centre has recruited over 100 subjects. Altogether 523 subjects have been randomised. The recruitment started after a pilot study in 1993 and was completed in May 1998. The study subjects were recruited through various methods, e.g. from epidemiological surveys and by opportunistic population screenings with special emphasis on the high-risk groups such as obese subjects and first-degree relatives of Type II diabetic patients. Subjects were also recruited through advertising in local newspapers.

The study subjects were randomly allocated to one of the two treatment modalities, the control group or the intensive diet and exercise intervention group. The randomisation was stratified by centre, sex and the mean 2 -h plasma glucose concentration (7.8-9.4 $\mathrm{mmol} / 1$ or $9.5-11.0 \mathrm{mmol} / \mathrm{l})$.

The main task of the study is to compare the cumulative incidence of diabetes among the intervention group with the cumulative incidence of diabetes in the control group. In addition we will evaluate the extent to which the cumulative incidence of diabetes is dependent on the observed changes in body weight, diet and physical activity.

Besides plasma glucose concentration we will monitor changes in several other CVD risk factors and cardiovascular morbidity and mortality in the study subjects. A logistic CVD risk score will be developed which will be based on the prospective follow-up of subjects with IGT in a random population sample [29]. We will consider the following variables when developing the CVD risk score: blood pressure, serum 
total and HDL cholesterol, serum triglycerides, serum insulin, body mass index (BMI), waist and hip circumferences, sagittal and abdominal diameters, smoking and plasma glucose. Variables that predict coronary heart disease or stroke independently will be included in the final model, which will be developed separately for men and women with IGT. Since the number of CVD events will presumably be too small during the study period to draw conclusions on this issue, we will continue monitoring the incidence of CVD complications (morbidity and mortality) after the primary study period. Furthermore, this study gives us the opportunity to evaluate the impact of diet and exercise intervention in subjects with different genetic backgrounds - those with an impaired insulin secretory capacity and those who are predominantly insulin resistant - applying the homeostasis model assessment method [30]. The ongoing search for genes for Type II diabetes will provide new information about the genetic markers of interest. We will be able to determine whether those subjects with IGT who progress to Type II diabetes share certain susceptibility genes and whether subjects with certain genotypes respond particularly well to intensive lifestyle interventions based on the results of genetic studies currently going on in Finland [31].

Inclusion and exclusion criteria. The diagnosis of diabetes and other categories of glucose intolerance were based on criteria adopted by the WHO in 1985 [32]. Overweight subjects $\left(\mathrm{BMI}>25 \mathrm{~kg} / \mathrm{m}^{2}\right)$ aged 40-64 years at randomisation and with IGT were eligible for the study. IGT was defined as a 2-h plasma glucose 7.8-11.0 mmol/l (OGTT $75 \mathrm{~g}$ ) with a non-diabetic fasting glucose concentration, i.e. plasma glucose less than $7.8 \mathrm{mmol} / \mathrm{l}$. After the first screening OGTT, a repeat OGTT was carried out in subjects with IGT and the mean value of the two 2-h glucose concentrations was used as the criterion for inclusion in the study. The inclusion criteria were developed during the recruitment period, but before the final criteria based on two OGTTs were decided, subjects were also enrolled based on one OGTT only ( $4 \%$ of the randomised subjects $)$, or based on high plasma glucose $(\geq 6.4 \mathrm{mmol} / \mathrm{l}$; fasting or random sample after a fast of at least $4 \mathrm{~h}$ ) together with one high 2-h plasma glucose concentration $(6 \%$ of the randomised subjects). All study subjects gave written informed consent.

These subjects were excluded: persons with a previous diagnosis of diabetes mellitus, other than gestational diabetes mellitus; persons involved regularly in a vigorous exercise programme as well as subjects receiving treatment to lower blood glucose, other than routine dietary and health advice; persons with any chronic disease making a 6-year survival improbable as well as other medical characteristics likely to interfere with participation in the study; subjects with unbalanced clinical conditions, such as thyroid and liver diseases, which could interfere with glucose metabolism.

Size of study population and power calculation. Based on the available prospective data from European populations the yearly incidence of diabetes among subjects with IGT varies between 1 and $10 \%$ and the 6-year cumulative incidence of diabetes in this high-risk group is thus estimated to be $35 \%$ [18]. Our preliminary evaluation of the presence of diabetes in the DPS subjects confirmed that this estimate is correct (data not shown). The DPS is designed to be large enough to detect a $35 \%$ reduction in diabetes incidence with an intensive diet and exercise intervention with $80 \%$ power (beta $=20 \%$ ) at the two-tailed $5 \%$ significance level (alpha $=5 \%)$, i.e. a reduction in cumulative incidence from $35 \%\left(\pi_{C}\right)$ to $22.8 \%$ $\left(\pi_{T}\right)$. We needed 3252 person years, which means that the study centres had to recruit 650 subjects to be followed for 5 years, or
542 subjects to be followed for 6 years. The possibility of a reduction in diabetes incidence by at least $35 \%$ is suggested by the results of the Malmöhus study, the Malmö feasibility study and the Da Qing study in which the effects of intervention were at least $40 \%[23,27,28]$. Furthermore, we are assuming that non-compliance in the DPS will not be worse than in these previous studies, making further adjustment for non-compliance unnecessary. The non-compliance used in the calculations was $10 \%$. The value used for $z$-alpha was 1.96 and z-beta 1.28 . The sample size calculation formula is shown below [33].

$n=\left[\frac{z_{\alpha} \sqrt{2 \pi(1-\pi)}+z_{\beta} \sqrt{\pi_{T}\left(1-\pi_{T}\right)+\pi_{C}\left(1-\pi_{C}\right)}}{\left(\pi_{T}-\pi_{C}\right)}\right]^{2}$

The cumulative incidence of diabetes will be calculated in the two groups per person years of follow-up using the intentionto-treat analysis. After randomisation the subjects will be followed until a notable difference in the rate of developing diabetes between the two randomisation groups exists, for approximately 6 years according to our sample size estimation.

\section{Intervention programme}

Control group. At the start of the trial a nutritionist advises the subjects in the control group to adjust total energy intake in order to reduce BMI below $25 \mathrm{~kg} / \mathrm{m}^{2}$ and to keep to a diet with less than $30 \%$ of daily energy from fat. Furthermore, they are advised to reduce alcohol intake and to stop smoking as appropriate. The dietary advice is provided by verbal and written information. Additional routine advice will be given to the subjects in the control group at their annual follow-up visits. Verbal general information about the health effects of recreational exercise is provided but no specific individual propositions and programmes are given.

Intervention group. A nutritionist gives frequent face-to-face dietary advice tailored to each subject individually and also in group sessions. The individually designed programme is planned to meet each subject's needs and interests and implemented taking into account the educational level of the subject. The person primarily in charge of preparing meals in the family, if different from the study subject, is also informed about the study aims and invited to join in the sessions with the nutritionist or the group meetings.

At the start of the trial the study physician and the nutritionist inform the subject about general risk factors for diabetes (e.g., role of obesity, sedentary life-style, genetics). Previous attempts to lose weight are discussed and a weight goal is established. The goal is a BMI of less than $25 \mathrm{~kg} / \mathrm{m}^{2}$ but, in practice, a weight loss of 5 to $10 \mathrm{~kg}$ depending on degree of obesity is the target for many study subjects.

The baseline 3-day food record is completed before the first appointment, and it forms the basis for the dietary advice during the second session. The intake of calories, fat, carbohydrates, protein, cholesterol and fibre are calculated and compared with recommendations. The subjects are advised to consume a diet with more than $50 \%$ of daily calories from carbohydrates; less than $10 \%$ from saturated fat and $20 \%$ from mono- and polyunsaturated fat, or up to $25 \%$ if the surplus is from monounsaturated fat [34]; cholesterol less than $300 \mathrm{mg} /$ day; and approximately $1.0 \mathrm{~g}$ protein per $\mathrm{kg}$ ideal body weight per day. The increase in the intake of dietary fibre to $15 \mathrm{~g}$ per $1000 \mathrm{kcal}$ or more, if tolerated, is encouraged. This goal is achieved by eating foods rich in natural fibre such as whole- 
meal products, vegetables, berries and fruit. One of the main goals in the dietary intervention is to reduce the intake of saturated fat and subjects are encouraged to use low-fat milk and milk products, low-fat meat and meat products, soft margarines and vegetable oil rich in monounsaturated fatty acids (primarily rapeseed oil).

After the randomisation, study visits are scheduled for 1-2 weeks, 5-6 weeks, 3, 4 and 6 months from the beginning of the study and thereafter every 3 months. Every three months 3-day food records are completed throughout the study. Weight is measured at every visit and the subject is asked about the lifestyle changes he or she has made. If no weight loss is observed, the energy content of the diet is re-evaluated. Eating-related behaviour is discussed and the nutrient intake based on food records is compared with recommendations.

If weight loss is not achieved during the first 6-12 months and the BMI is over $30 \mathrm{~kg} / \mathrm{m}^{2}$, a very low calorie diet (VLCD) is considered. During a 6-12 week VLCD phase, regular group meetings (at intervals of 1-2 weeks) are held.

The subjects in the intervention group are individually guided to increase their physical activity. Exercise programmes differ between study centres according to local situation and facilities. Endurance exercise (walking, jogging, swimming, aerobic ball games, skiing) is recommended to increase aerobic capacity and cardiorespiratory fitness. Supervised, progressive, individually tailored circuit-type resistance training sessions are organised, if possible, twice a week. The moderate intensity and medium- to high-volume programmes are designed to improve the functional capacity and strength of the large muscle groups of the upper and lower body. Compliance with the intensive diet-exercise programme is monitored by individual interviews at each clinical visit.

Follow-up and outcome indices. The primary outcome under study is the development of diabetes. All study subjects have an OGTT at each annual visit. If the WHO criteria for diabetes are met, diabetes must be confirmed by another OGTT at least 1 week after the first one.

The secondary outcomes under study are changes in plasma glucose, serum insulin and glycated haemoglobin $\left(\mathrm{HbA}_{1 \mathrm{c}}\right)$. Additional outcomes are changes in the other cardiovascular risk factors, i.e. blood pressure, serum lipids and uric acid. Testing for the secondary outcomes is done once a year. Cardiovascular mortality and morbidity will be monitored through the nation-wide death registry and hospital discharge registry and will continue also after the study period is over.

In addition to the comparison of outcomes between the groups according to treatment, the clinical course and prognosis within treatment groups are assessed. In this respect, the group assigned to the routine diet-exercise programme will be of primary interest because it is this group that will provide information on the natural history of IGT. Post-hoc analyses will also be carried out to assess the effect of actually observed lifestyle changes on the primary and secondary outcomes.

Examinations. A medical history and a physical examination is done on a yearly basis. Anthropometric measurements include height, weight, waist and hip circumferences and sagittal and transverse diameters. Waist circumference is measured midway between the lowest rib and iliac crest and hip circumference over the great trochanters, with $0.5 \mathrm{~cm}$ precision with the subject in a standing position. Sagittal and transverse diameters, measured with the subject lying on his or her back on a hard surface, are taken as the distance from the surface to the highest point of the abdomen (sagittal diameter) and the maximum width of the abdomen (transverse diameter) at the level of the iliac crest, with $0.1 \mathrm{~cm}$ precision using specially built equipment. Lean body mass is assessed using near-infrared spectroscopy equipment (Futrex, Gaithersburg, Md., USA) or bio-impedance equipment (RJL Systems, Detroit, Mich., USA).

A 12-lead supine resting ECG is recorded annually and coded using the Minnesota code [35]. Blood pressure is measured yearly on the right arm with the subject in a sitting position and is measured a second time, after 10 min of rest, using a standard sphygmomanometer. The following laboratory assessments are made: serum cholesterol, HDL-cholesterol, triglycerides, creatinine and uric acid. Liver function test (serum gamma-glytamyl transferase) and thyroid stimulating hormone levels are measured as well. A 2-h OGTT is done to determine glucose tolerance. Samples for glucose and insulin are taken at baseline, $30 \mathrm{~min}, 60 \mathrm{~min}$ and $120 \mathrm{~min}$. Samples for the measurements of antibodies to glutamic acid decarboxylase (GAD) and typing of human leukocyte antigens (HLA), and the determination of haemostatic factors (fibrinogen and plasminogen activator inhibitor-1) are also obtained.

Plasma glucose and $\mathrm{HbA}_{1 \mathrm{c}}$ are determined locally according to standard guidelines. All other biochemical determinations are done in the central laboratory of the Department of Biochemistry, National Public Health Institute, Helsinki. The haemostatic factors are analysed in the laboratory of Research and Development Centre of the Social Insurance Institution, Turku. Serological typing of HLA class I antigens and molecular typing of HLA class II antigens are done in the central laboratory at the National Public Health Institute, Helsinki. DNA has been extracted from every subject and kept frozen for additional molecular genetic analyses to be done in the future.

Serum insulin is determined with a radioimmunoassay (Pharmacia, Uppsala, Sweden). The intra-assay CV is $5.3 \%$ and interassay CV 7.6\%. Serum total cholesterol, HDL-cholesterol and triglycerides are determined using an enzymatic assay method (CHOD-PAP, Boehringer Mannheim, Germany, Monotest). The intra-assay CV for total cholesterol, HDLcholesterol and triglycerides are 1.0, 1.3 and $1.1 \%$, respectively, and the interassay CV 1.2, 2.2 and $1.5 \%$, respectively.

Plasma glucose concentrations determined during the follow-up will be given to the participants at the end of the study only, except in cases where frank diabetes is diagnosed.

A KIHD (Kuopio Ischemic Heart Disease Study) 24-h exercise diary is kept every 3 months and a KIHD 12-month leisure physical activity history is completed on annual visits [36]. A 2-km walking test to determine the physical fitness index is done on a yearly basis [37, 38]. A 3-day food record is kept before randomisation and thereafter annually (control group) or every 3 months (intervention group). Portion sizes for food are estimated using a validated [39] portion size picture booklet [40]. Intake of nutrients is computed using the programme developed at the National Public Health Institute [41]. The analyses of nutrient intake for the intervention and control groups are based on the annual food records.

The health status of the subjects is measured annually using RAND 36-Item Health Survey 1.0 [42].

Statistical analysis. A two-tailed paired $t$ test was used to analyse the differences within the groups at baseline and at year 1 , and an unpaired $t$ test to analyse the difference in mean change from baseline to year 1 between the intervention and control groups. Mean changes and their $95 \%$ confidence intervals were calculated. A $p$ value less than 0.05 for the differences between the mean values is considered statistically significant. The analyses were done using SAS software (SAS Institute, Cary, NC, USA). 
Table 1. Clinical and metabolic characteristics of the study population $(n=212)$ at baseline. Values are given as means \pm SD

\begin{tabular}{lcc}
\hline Parameter & $\begin{array}{c}\text { Intervention } \\
\text { group }\end{array}$ & Control group \\
\hline Number of subjects (men/women) & $112(44 / 68)$ & $100(33 / 67)$ \\
Age (years) & $54 \pm 7$ & $52 \pm 7$ \\
BMI $\left(\mathrm{kg} / \mathrm{m}^{2}\right)$ & $31.3 \pm 5.0$ & $31.1 \pm 4.6$ \\
Fat mass $(\mathrm{kg})$ & $30.3 \pm 9$ & $30.2 \pm 8$ \\
Waist circumference $(\mathrm{cm})$ & $102.6 \pm 11.6$ & $100.5 \pm 10.2$ \\
Hip circumference $(\mathrm{cm})$ & $111.0 \pm 11.8$ & $109.7 \pm 9.3$ \\
Sagittal diameter $(\mathrm{cm})$ & $26.1 \pm 4.2$ & $25.9 \pm 4.5$ \\
Transverse diameter $(\mathrm{cm})$ & $38.7 \pm 4.7$ & $38.2 \pm 4.4$ \\
Fasting plasma glucose $(\mathrm{mmol} / \mathrm{l})$ & $6.0 \pm 0.8$ & $6.1 \pm 0.7$ \\
2-h plasma glucose $(\mathrm{mmol} / \mathrm{l})$ & $8.7 \pm 1.2$ & $8.8 \pm 1.3$ \\
Fasting serum insulin $(\mathrm{pmol} / \mathrm{l})$ & $114 \pm 53$ & $122 \pm 99$ \\
2-h serum insulin $(\mathrm{pmol} / \mathrm{l})$ & $707 \pm 479$ & $669 \pm 380$ \\
Serum total cholesterol $(\mathrm{mmol} / \mathrm{l})$ & $5.5 \pm 0.9$ & $5.7 \pm 1.0$ \\
Serum HDL-cholesterol $(\mathrm{mmol} / \mathrm{l})$ & $1.15 \pm 0.29$ & $1.18 \pm 0.26$ \\
Serum triglycerides $(\mathrm{mmol} / \mathrm{l})$ & $1.66 \pm 0.78$ & $1.85 \pm 0.82$ \\
Systolic blood pressure $(\mathrm{mm} \mathrm{Hg})$ & $139 \pm 18$ & $136 \pm 19$ \\
Diastolic blood pressure $(\mathrm{mm} \mathrm{Hg})$ & $85 \pm 10$ & $86 \pm 11$ \\
\hline
\end{tabular}

Table 2. Clinical and metabolic characteristics of the study population $(n=212)$ after 1 year. Values are given as means \pm SD

\begin{tabular}{|c|c|c|}
\hline Parameter & $\begin{array}{l}\text { Intervention } \\
\text { group }\end{array}$ & Control group \\
\hline $\begin{array}{l}\text { BMI }\left(\mathrm{kg} / \mathrm{m}^{2}\right) \\
\text { Fat mass }(\mathrm{kg}) \\
\text { Waist circumference }(\mathrm{cm}) \\
\text { Hip circumference }(\mathrm{cm}) \\
\text { Sagittal diameter }(\mathrm{cm}) \\
\text { Transverse diameter }(\mathrm{cm})\end{array}$ & $\begin{aligned} 29.6 & \pm 4.8 \\
27.8 & \pm 9 \\
99.2 & \pm 11.5 \\
107.5 & \pm 10.6 \\
23.3 & \pm 3.2 \\
37.5 & \pm 4.2\end{aligned}$ & $\begin{array}{c}30.8 \pm 4.6 \\
29.8 \pm 8 \\
100.5 \pm 10.7 \\
109.1 \pm 9.4 \\
24.5 \pm 3.5^{1} \\
38.2 \pm 4.3\end{array}$ \\
\hline $\begin{array}{l}\text { Fasting plasma glucose }(\mathrm{mmol} / \mathrm{l}) \\
\text { 2-h plasma glucose }(\mathrm{mmol} / \mathrm{l}) \\
\text { Fasting serum insulin }(\mathrm{pmol} / \mathrm{l}) \\
\text { 2-h serum insulin }(\mathrm{pmol} / \mathrm{l})\end{array}$ & $\begin{array}{c}5.9 \pm 0.7 \\
7.8 \pm 1.8 \\
84 \pm 30 \\
509 \pm 342\end{array}$ & $\begin{aligned} 6.4 & \pm 0.8^{2} \\
8.5 & \pm 2.3^{2} \\
99 & \pm 46 \\
593 & \pm 388\end{aligned}$ \\
\hline $\begin{array}{l}\text { Serum total cholesterol }(\mathrm{mmol} / \mathrm{l}) \\
\text { Serum HDL-cholesterol }(\mathrm{mmol} / \mathrm{l}) \\
\text { Serum triglycerides }(\mathrm{mmol} / \mathrm{l})\end{array}$ & $\begin{array}{l}5.4 \pm 0.8 \\
1.24 \pm 0.29 \\
1.39 \pm 0.47\end{array}$ & $\begin{array}{l}5.6 \pm 1.0 \\
1.20 \pm 0.25 \\
1.83 \pm 0.87^{2}\end{array}$ \\
\hline $\begin{array}{l}\text { Systolic blood pressure }(\mathrm{mm} \mathrm{Hg}) \\
\text { Diastolic blood pressure }(\mathrm{mm} \mathrm{Hg})\end{array}$ & $\begin{array}{r}133 \pm 18 \\
81 \pm 10\end{array}$ & $\begin{aligned} 134 & \pm 17 \\
85 & \pm 10^{1}\end{aligned}$ \\
\hline
\end{tabular}

$\overline{{ }^{1} p<0.05 ;{ }^{2} p<0.001 \text {, intervention group versus control group }}$

\section{Results}

This paper presents results for the first 212 subjects who have participated in the study for at least 1 year and thus have baseline and 1-year results available. The baseline characteristics in both groups were similar with regard to age, plasma glucose and serum insulin concentrations, degree of obesity, blood pressure and lipid values (Table 1). Baseline fasting (6.0 vs $6.1 \mathrm{mmol} / \mathrm{l})$ and 2 -h plasma glucose (8.7 vs $8.8 \mathrm{mmol} / \mathrm{l}$ ) concentrations were similar in both groups. After one year, however, both fasting plasma glucose (5.9 vs $6.4 \mathrm{mmol} / \mathrm{l} ; p<0.001)$ and 2-h plasma glucose $(7.8$ vs $8.5 \mathrm{mmol} / \mathrm{l} ; p<0.05)$ were significantly lower in the intervention group (Table 2).

At the 1-year examination (Table 3), body weight had decreased by $4.7 \mathrm{vs} 0.9 \mathrm{~kg}$ in the intervention and control group, respectively $(p<0.001)$. The anthropometric measurements of the intervention group had also improved compared with the control group. The lipid profile tended to have improved more in the intervention group than in the control group; serum triglyceride concentration had decreased by $0.21 \mathrm{mmol} / 1$ in the intervention group whereas no changes had occurred in the control group $(p=0.04)$. Both groups showed a decrease in blood pressure, this was significant only in the intervention group. The differences in the changes between the groups in both systolic blood pressure $(p<0.05)$ and diastolic blood pressure were statistically significant $(p<0.05)$.

\section{Discussion}

Primary prevention of Type II diabetes was proposed as early as the 1920s when Joslin made a strong plea to diabetes researchers about prevention of the disease [19]. The present evidence suggests that a change in lifestyle might reduce progression from IGT to Type II diabetes [27, 28, 43]. This interim report from the DPS clearly shows that lifestyle intervention is feasible and effective in Finnish subjects with IGT.

Several of the previous studies focusing on the prevention of Type II diabetes in individuals with IGT used pharmacological intervention, i.e. sulphonylureas and biguanides usually combined with dietary advice. Theoretically, sulphonylureas can affect both insulin secretion and insulin sensitivity and there are some encouraging results $[17,21,22,25]$. Also, the biguanides theoretically offer the potential to improve glucose tolerance in individuals at risk of Type II diabetes. Despite these considerations and positive findings, neither the Bedford (sulphonylurea) nor Whitehall (biguanides) studies in England established that either diet or oral antidiabetic agents have a discernible effect on the subsequent incidence of diabetes $[24,26]$. On the other hand, both the Malmöhus study and the Malmö feasibility study in Sweden suggest the possibility of a reduction in diabetes incidence by at least $30 \%$ through intervention [23, 27, 44]. In the Malmöhus study in Sweden, persons with IGT were randomly assigned to one of three groups, all of which received diet treatment. In two of the groups the patients also received tablets, either tolbutamide or a placebo. The overall incidence of overt diabetes was $12 \%$ in the study, with no statistically significant differences between the groups. In the tolbutamide group, however, the diabetes incidence was $10 \%$, and among those who did not receive any medication 
Table 3. The mean changes \pm SD and $95 \%$ confidence intervals $(95 \% \mathrm{CI})$ in clinical and metabolic parameters assessed

\begin{tabular}{|c|c|c|c|c|}
\hline & \multicolumn{2}{|c|}{ Intervention group } & \multicolumn{2}{|l|}{ Control group } \\
\hline & change $\pm \mathrm{SD}$ & $(95 \% \mathrm{CI})$ & change $\pm \mathrm{SD}$ & $(95 \% \mathrm{CI})$ \\
\hline $\begin{array}{l}\text { Weight }(\mathrm{kg}) \\
\text { Fat mass }(\mathrm{kg}) \\
\text { Waist circumference }(\mathrm{cm}) \\
\text { Hip circumference }(\mathrm{cm}) \\
\text { Sagittal diameter }(\mathrm{cm}) \\
\text { Transverse diameter }(\mathrm{cm})\end{array}$ & $\begin{array}{l}-4.7 \pm 5.5 \\
-2.5 \pm 3.9 \\
-3.5 \pm 5.1 \\
-3.5 \pm 4.8 \\
-2.8 \pm 3.4 \\
-1.0 \pm 2.2\end{array}$ & $\begin{array}{l}(-5.8 ;-3.7) \\
(-3.4 ;-1.7) \\
(-4.4 ;-2.5) \\
(-4.4 ;-2.6) \\
(-3.4 ;-2.1) \\
(-1.5 ;-0.6)\end{array}$ & $\begin{array}{r}-0.9 \pm 4.1^{3} \\
-0.3 \pm 3.3^{3} \\
0.0 \pm 4.5^{3} \\
-0.6 \pm 3.3^{3} \\
-1.5 \pm 4.0^{1} \\
-0.1 \pm 1.8^{3}\end{array}$ & $\begin{array}{l}(-1.7 ; 0.0) \\
(-1.1 ; 0.4) \\
(-0.9 ; 0.9) \\
(-1.3 ; 0.0) \\
(-2.3 ;-0.7) \\
(-0.4 ; 0.3)\end{array}$ \\
\hline $\begin{array}{l}\text { Serum total cholesterol }(\mathrm{mmol} / \mathrm{l}) \\
\text { Serum HDL-cholesterol }(\mathrm{mmol} / \mathrm{l}) \\
\text { Serum triglycerides }(\mathrm{mmol} / \mathrm{l})\end{array}$ & $\begin{array}{r}-0.2 \pm 0.6 \\
0.05 \pm 0.17 \\
-0.21 \pm 0.61\end{array}$ & $\begin{array}{l}(-0.36 ;-0.11) \\
(0.02 ; 0.09) \\
(-0.34 ;-0.08)\end{array}$ & $\begin{array}{l}-0.1 \pm 0.7 \\
0.01 \pm 0.15 \\
0.00 \pm 0.69^{1}\end{array}$ & $\begin{array}{l}(-0.26 ; 0.06) \\
(-0.03 ; 0.04) \\
(-0.16 ; 0.15)\end{array}$ \\
\hline
\end{tabular}

${ }^{1} p<0.05$ vs intervention group; ${ }^{2} p<0.01$ vs intervention group; ${ }^{3} p<0.001$ vs intervention group

it was $13 \%$. The data were also analysed according to estimates of compliance and none of those who continued to take tolbutamide developed diabetes [23].

The feasibility of lifestyle intervention in the prevention of Type II diabetes was shown more recently in a study of 217 middle-aged Swedish men with IGT [27]. The men were divided into two groups: 161 were treated with diet and exercise and 56 formed a reference group. The groups were not assigned at random. There was a significant weight loss in the active intervention group, most of which was maintained for 5 years, and no weight loss in the reference group. After 5 years, $11 \%$ of the intervention group and $21 \%$ of the reference group had developed diabetes according to WHO criteria. Thus the incidence in the treated group was $50 \% \quad(95 \%$ $\mathrm{CI}=0.3-1.0)$ lower than in the reference group [27]. A treatment effect was not unequivocally established because the study groups were not assigned at random, and the subjects themselves could decide whether they wanted to take part in the lifestyle programmes or not.

Recently, data on the preventive effects of diet and exercise have been reported in a cluster-randomised clinical trial on 577 subjects with IGT in Da-Qing, China [28]. The cumulative 6-year incidence of Type II diabetes was notably lower in each of the three active intervention groups (41.1-46.0\%) compared with the control group $(67.7 \%)$. The overall cumulative 6-year incidence of diabetes was very high in all study groups, and most of the individuals studied could not be considered as "typical" high-risk obese IGT subjects according to a European standard.

Thus, the question of the prevention of Type II diabetes by drugs or lifestyle intervention is not completely settled, and there is an urgent need to carry out well-designed trials on the prevention of Type II diabetes in high-risk subjects such as people with IGT.

An intervention programme using lifestyle intervention alone is a natural way of preventing Type II diabetes since the increased prevalence and incidence of the disease in most European countries is mainly due to the adaptation of a sedentary lifestyle and excessive food intake. At present a few large-scale intervention studies on the prevention of Type II diabetes are being done using pharmaceutical compounds $[45,46]$. Compared with drug intervention one major advantage is that no pharmacological side effects are expected to occur with diet-exercise intervention. Furthermore, a non-pharmacological approach is not only rational with regard to current knowledge of the risk factors of Type II diabetes, but this approach can also reduce the risk of atherosclerotic vascular disease common in IGT and Type II diabetes [11, 47, 48]. Both diet and exercise are known to reduce the degree of insulin resistance $[49,50]$. Furthermore the Oslo Diet and Exercise Study on insulin resistance showed that the combination of diet and exercise was most effective in reversing the insulin resistance syndrome compared with each of these components separately [49].

In the DPS special emphasis has been put on the intervention programme. The nutritional intervention incorporates elements from several theoretical models, e.g. client-centred counselling (individual sessions with the nutritionist) and social support (incorporating spouse into the study). Also, the greater frequency of interaction with the participants has been considered important to increase the level of support. In a study on IGT subjects receiving both dietary and exercise advice for a 6-month period, it was observed that an intervention programme of this length was not sufficient to induce permanent life- 
style changes [51]. On the other hand, it has been shown in patients with newly diagnosed Type II diabetes that even a 1-year intervention programme significantly improves weight loss, metabolic control and cardiovascular risk factors compared with conventional treatment programmes [52].

The preliminary 1-year results of the Finnish DPS prove the feasibility of lifestyle intervention in persons with IGT. The positive findings regarding changes in body weight, other anthropometric measures and metabolic parameters are encouraging.

The difference in plasma glucose concentrations between the groups was striking. Even during the first year there was some deterioration in fasting glucose in the control group. In the 2-h plasma glucose concentration an improvement in both groups was noted but the change was significant only in the intervention group. The mean value of the 2 -h plasma glucose concentration at the 1-year examination of the intervention group, i.e. $7.8 \mathrm{mmol} / \mathrm{l}$, was only minimally above the normal concentration, according to the current criteria of the WHO.

The IGT subjects had fairly normal lipid values. Since baseline lipid levels were mostly normal, the positive changes in HDL-cholesterol and triglycerides can be taken as indicators of the success of the intervention programme. These changes could also be beneficial on a long-term basis since atherosclerotic vascular disease in IGT and Type II diabetes has been shown to be associated with low HDL-cholesterol and high triglyceride levels [50, 53].

Regardless of the outcome measures for obesity, the intervention programme proved successful. Overall body weight decreased, although the average BMI was still high at 1 year. In particular abdominal obesity and visceral fat are strongly associated with cardiovascular morbidity $[54,55]$. The decrease in abdominal obesity in the intervention group is therefore important. Data from studies on the effect of lifestyle changes on newly diagnosed Type II diabetic patients show that weight reduction appears to be the major determinant of beneficial outcome measures.

The great improvement in the metabolic changes in the intervention group supports a successful continuation of the study, if lifestyle changes remain permanent, and the onset of Type II diabetes could be postponed by several years. Whether this happens, will be seen in the future as the DPS now has all its study subjects recruited and the programme is in full operation. A detailed report on the baseline characteristics on all subjects randomised will be provided in a separate report.

We want to stress that this kind of lifestyle programme, which in our present study is targeted to prevent Type II diabetes, is similar to the recommendations for the prevention of cardiovascular disease, many forms of cancer and osteoporosis and can thus be extended to cover much more than diabetes alone. This is of the utmost importance since part of the association between IGT and CVD is due to the higher levels of CVD risk factors in IGT subjects. Since subjects with IGT are at increased risk for CVD, treatment strategies should be directed at both CVD and Type II diabetes prevention.

Acknowledgements. This study has been partly funded by the Academy of Finland, Ministry of Education, Novo Nordisk Foundation, Yrjö Jahnsson Foundation and the Finnish Diabetes Research Foundation.

\section{References}

1. Kannel WB, McGee DL (1979) Diabetes and cardiovascular disease. The Framingham study. JAMA 241: 2035-2038

2. Fuller JH, Shipley MJ, Rose G, Jarrett RJ, Keen H (1983) Mortality from coronary heart disease and stroke in relation to degree of glycaemia: the Whitehall Study. BMJ 287: 867-870

3. Manson JE, Colditz GA, Stampfer MJ et al. (1991) A prospective study of maturity-onset diabetes mellitus and risk of coronary heart disease and stroke in women. Arch Intern Med 151: 1141-1147

4. Nathan DM (1993) Long-term complications of diabetes mellitus. N Engl J Med 328: 1676-1685

5. Stamler J, Vaccaro O, Neaton J, Wentworth D, for the Multiple Risk Factor Intervention Trial Research Group (1993) Diabetes, other risk factors, and 12-yr cardiovascular mortality for men screened in the Multiple Risk Factor Intervention Trial. Diabetes Care 16: 434-444

6. Rubin RJ, Altman WM, Mendelson DN (1994) Health care expenditures for people with diabetes mellitus. J Clin Endocrinol Metab 78: 809A-809F

7. Kangas T, Aro S, Koivisto VA et al. (1996) Structure and costs of health care of diabetic patients in Finland. Diabetes Care 19: 494-497

8. UK Prospective Diabetes Study Group (1998) Tight blood pressure control and risk of macrovascular and microvascular complications in type 2 diabetes: UKPDS 38. BMJ 317: 703-713

9. UK Prospective Diabetes Study Group (1998) Intensive blood-glucose control with sulphonylureas or insulin compared with conventional treatment and risk of complications in patients with type 2 diabetes (UKPDS 33). Lancet 352: 837-853

10. Amos AF, McCarty DJ, Zimmet P (1997) The rising global burden of diabetes and its complications: Estimates and projections to the year 2010. Diabet Med 14: S7-S85

11. Tuomilehto J, Tuomilehto-Wolf E, Zimmet P, Alberti KGMM, Knowler WC (1997) Primary prevention of diabetes mellitus. In: Alberti KGMM, Zimmet P, DeFronzo RA, Keen H (eds) International Textbook of Diabetes Mellitus, 2nd edn. Wiley, Chichester New York Brisbane Toronto, pp 1799-1827

12. Hamman RF (1992) Genetic and environmental determinants of non-insulin-dependent diabetes mellitus (NIDDM). Diabetes Metab Rev 8: 287-338

13. Eriksson J, Franssila-Kallunki A, Ekstrand A et al. (1989) Early metabolic defects in persons at increased risk for non-insulin-dependent diabetes mellitus. N Engl J Med 321: $337-343$ 
14. Warram JH, Martin BC, Krolewski AS, Soeldner JS, Kahn CR (1990) Slow glucose removal rate and hyperinsulinemia precede the development of type II diabetes in the offspring of diabetic parents. Ann Intern Med 113: 909 915

15. Harris MI (1989) Impaired glucose tolerance in the U.S. population. Diabetes Care 12: 464-474

16. Stengård JH, Tuomilehto J, Pekkanen J et al. (1992) Diabetes mellitus, impaired glucose tolerance and mortality among elderly men: The Finnish cohorts of the Seven Countries Study. Diabetologia 35: 760-765

17. Knowler WC, Sartor G, Melander A, Schersten B (1997) Glucose tolerance and mortality, including a substudy of tolbutamide treatment. Diabetologia 40: 680-686

18. Valle T, Tuomilehto J, Eriksson J (1997) Epidemiology of NIDDM in Europids. In: Alberti KGMM, Zimmet P, DeFronzo RA, Keen H (eds) International textbook of diabetes mellitus, 2nd edn. Wiley, Chichester New York Brisbane Toronto, pp 125-142

19. Joslin EP (1921) The prevention of diabetes mellitus. JAMA 76: 79-84

20. World Health Organisation (1994) Primary prevention of diabetes mellitus. Technical Report Series No 844, WHO, Geneva

21. Fajans SS, Conn JW (1960) Tolbutamide-induced improvement in carbohydrate tolerance of young people with mild diabetes mellitus. Diabetes 9: 83-88

22. Paasikivi J (1970) Long-term tolbutamide treatment after myocardial infarction. Acta Med Scand; [Suppl] 507

23. Sartor G, Schersten B, Carlstrom S, Melander A, Norden A, Persson G (1980) Ten-year follow-up of subjects with impaired glucose tolerance: prevention of diabetes by tolbutamide and diet regulation. Diabetes 29: 41-49

24. Keen H, Jarrett RJ, McCartney P (1982) The ten-year follow-up of the Bedford survey (1962-1972): glucose tolerance and diabetes. Diabetologia 22: 73-78

25. Ratzmann KP, Witt S, Schulz B (1983) The effect of longterm glibenclamide treatment on glucose tolerance, insulin secretion and serum lipids in subjects with impaired glucose tolerance. Diabetes Metab 9: 87-93

26. Jarrett RJ, Keen H, McCartney P (1984) The Whitehall study: ten year follow-up report on men with impaired glucose tolerance with reference to worsening to diabetes and predictors of death. Diabet Med 1: 279-283

27. Eriksson KF, Lindgärde E (1991) Prevention of Type 2 (non-insulin-dependent) diabetes mellitus by diet and physical exercise: The 6-year Malmö feasibility study. Diabetologia 34: 891-898

28. Pan X-P, Li G-W, Hu Y-H et al. (1997) Effects of diet and exercise in preventing NIDDM in people with impaired glucose tolerance. The Da Qing IGT and Diabetes Study. Diabetes Care 20: 537-544

29. Tuomilehto J, Korhonen HJ, Kartovaara L et al. (1991) Prevalence of diabetes mellitus and impaired glucose tolerance in the middle-aged population of three areas in Finland. Int J Epidemiol 20: 1010-1017

30. Matthews DR, Hosker JP, Rudenski AS et al. (1985) Homeostasis model assessment: insulin resistance and betacell function from fasting plasma glucose and insulin concentrations in man. Diabetologia 28: 412-419

31. Valle T, Tuomilehto J, Bergman RN et al. (1998) Mapping genes for NIDDM. Design of the Finland-United States Investigation of NIDDM Genetics (FUSION) Study. Diabetes Care 21: 949-958

32. World Health Organization Expert Committee (1985) Diabetes Mellitus. Technical Report Series No 742, WHO, Geneva
33. Norman GR, Streiner DL (1994) Biostatistics. The bare essentials. Mosby, St Louis Baltimore Boston Chicago London Madrid Philadelphia Sydney Toronto

34. Sarkkinen E, Schwab U, Niskanen L et al. (1996) The effects of monounsaturated-fat enriched diet and polyunsaturated-fat enriched diet on lipid and glucose metabolism in subjects with impaired glucose tolerance. Eur J Clin Nutr 50: 592-598

35. Prineas RJ, Crow RS, Blackburn H (1982) The Minnesota code manual of eloectrocardiographic findings. Laboratory of Physiological Hygiene, School of Public Health, University of Minnesota

36. Lakka T (1994) Leisure time physical activity, cardiorespiratory fitness, biological coronary risk factors and coronary heart disease: a population study in men in eastern Finland. Academic Doctoral Dissertation. Kuopio, Finland. Kuopio University Printing Office

37. Oja P, Laukkanen R, Pasanen M, Tyry T, Vuori I (1991) A 2-km walking test for assessing the cardiorespiratory fitness of healthy adults. Int J Sports Med 12:356-362

38. Laukkanen R, Oja P, Pasanen M, Vuori I (1992) Validity of a two kilometre walking test for estimating maximal aerobic power in overweight adults. Int J Obes Relat Metab Disord 16: 263-268

39. Pietinen P, Hartman AM, Haapa E et al. (1988) Reproducibility and validity of dietary assessment instruments. I. A self-administered food use questionnaire with a portion size picture booklet. Am J Epidemiol 128: 655-666

40. Haapa E, Toponen T, Pietinen P, Räsänen L (1985) Annoskuvakirja. Painokaari Oy, Helsinki

41. Ovaskainen M-L, Valsta L, Lauronen J (1996) The compilation of food analysis values as a database for dietary studies - the Finnish experience. Food Chemistry 57: 133-136

42. Hays RD, Donald Sherbourne C, Mazel RM (1993). The RAND 36-Item Health Survey 1.0. Health Econ 2: 217-227

43. Bourn D, Mann J, McSkimming B, Waldron M, Wishart J (1994) Impaired glucose tolerance and NIDDM: does a lifestyle intervention program have an effect? Diabetes Care 17: 1311-1319

44. Knowler WC, Narayan KM, Hanson RL et al. (1995) Preventing non-insulin-dependent diabetes. Diabetes 44: 483-488

45. Chiasson J-L, Josse RG, Leiter LA et al. (1995) Can we prevent the development of non-insulin-dependent diabetes mellitus (abstract). Diabetes 44 [Suppl 1]:62 A

46. National Institutes of Health (1993) Non-insulin dependent diabetes primary prevention trial. NIH guide grants contratcs 22: 1-20

47. Page RCL, Harnden KE, Walravens NKN et al. (1993) 'Healthy living' and sulphonylurea therapy have different effects on glucose tolerance and risk factors for vascular diseases in subjects with impaired glucose tolerance QJM 86: $145-154$

48. Alberti KGMM (1996) The clinical implications of impaired glucose tolerance. Diabet Med 13: 927-937

49. Torjesen PA, Birkeland KI, Anderssen SA, Hjermann I, Holme I, Urdal P (1997). Lifestyle changes may reverese development of the insulin resistance syndrome. Diabetes Care 20: 26-31

50. Uusitupa MIJ (1996) Early lifestyle intervention in patients with non-insulin-dependent diabetes mellitus and impaired glucose tolerance. Ann Med 28: 445-449

51. Page RCL, Harnden KE, Cook JTE, Turner RC (1992) Can life-styles of subjects with impaired glucose tolerance be changed? A feasibility study. Diabet Med 9: 562566 
52. Laitinen JH, Ahola IE, Sarkkinen ES, Winberg RL, Harmaakorpi-Iivonen PA, Uusitupa MI (1993) Impact of intensified dietary therapy on energy and nutrient intakes and fatty acid composition of serum lipids in patients with recently diagnosed non-insulin-dependent diabetes mellitus. J Am Diet Assoc 93: 276-283

53. Uusitupa MIJ, Niskanen LK, Siitonen O, Voutilainen E, Pyörälä K (1993) Ten-year cardiovascular mortality in relation to risk factors and abnormalities in lipoprotein compo- sition in type 2 (non-insulin-dependent) diabetic and nondiabetic subjects. Diabetologia 36: 1175-1184

54. Vague J (1956) Degree of masculine differentiation of obesities: factor determinating predisposition to diabetes, atherosclerosis, gout, and uric calculous disease. Am J Clin Nutr 4: 220-234

55. Pi-Sunyer FX (1993) Medical hazards of obesity. Ann Intern Med 119: 655-660 